\section{Neoadjuvante Blasenkrebstherapie auf evidenzbasierte Füße stellen}

Eine Metaanalyse fand für die adjuvante Chemotherapie bei muskelinvasivem Blasenkarzinom (MIBC, pT2-4a) nur schwache Hinweise auf einen Überlebenszeitgewinn, während neoadjuvante Cisplatin-basierte Kombinations-Chemotherapien immerhin einen absoluten Vorteil von $5 \%$ bezüglich des Gesamtüberlebens erreichen konnten. Randomisiert-kontrollierte Studien dazu fehlen. Eine retrospektive Analyse versuchte jetzt, einen ersten Schritt von der erfahrungsbasierten zur evidenzbasierten neoadjuvanten Therapie zu machen.

\begin{abstract}
A ls dosisintensives Regime kann die akzelierte neoadjuvante Therapie mit Methotrexat, Vinblastin, Doxorubicin und Cisplatin (AMVAC) die Zeit bis zur definitiven, potenziell kurativen Therapie gering halten. Die Autoren analysierten retrospektiv Wirksamkeit und Toxizität einer neoadjuvanten Chemotherapie mit AMVAC bei 80 konsekutiven Patienten mit MIBC und die längerfristigen Auswirkungen auf den Gesamterfolg der Therapie für die Patienten. AMVAC wurde vor einer radikalen chirurgischen oder strahlentherapeutischen Therapie in drei bis vier Zyklen in zweiwöchigen Intervallen verabreicht.
\end{abstract}

Die Dosierungen lagen für Methotrexat bei $30 \mathrm{mg} / \mathrm{m}^{2}$, für Vinblastin bei $3 \mathrm{mg} /$ $\mathrm{m}^{2}$, für Doxorubicin bei $30 \mathrm{mg} / \mathrm{m}^{2}$ und für Cisplatin bei $70 \mathrm{mg} / \mathrm{m}^{2}$. Die Chemotherapie wurde unterstützt durch die Gabe von Granulozyten-Kolonie-stimulierendem Faktor (GCS-F).

Bei $84 \%$ der Patienten konnte die vorgesehene Therapie in der geplanten Zeit und Dosis durchgeführt werden. Letztlich haben sogar alle 80 Patienten die geplante neoadjuvante Therapie erhalten. Von $42 \%$ der Patienten lagen Toxizitätsdaten vor. Bei $26 \%$ von ihnen wurden Toxizitäten des Grads 3 und 4 dokumentiert, davon $12 \%$ Grad-3- oder -4-Neu- ropenien. Bei keinem Patienten musste infolge der neoadjuvanten Therapie auf die potenziell kurative definitive Therapie verzichtet werden.

Ein pathologisch bestätigtes komplettes Ansprechen auf die neoadjuvante AMVAC-Therapie zeigte sich bei $43 \%$ der 60 chirurgisch behandelten Patienten, in objektives radiologisches lokales Ansprechen bei $83 \%$ der 57 Patienten, für die auswertbare Daten vorlagen. $65 \%$ der Patienten überlebten zwei Jahre krankheitsfrei. Die 2-Jahres-Gesamtüberlebensrate lag bei $77 \%$.

Fazit: Nach der retrospektiven Auswertung kann AMVAC sicher als neoadjuvante Therapie des MIBC eingesetzt werden. Dabei waren die pathologischen und radiologischen Ansprechraten viel versprechend. Daher empfehlen die $\mathrm{Au}-$ toren diese Therapie als geeignetes Regime für zukünftige randomisierte, kontrollierte Studien.

Friederike Klein

Blick $C$ et al. Accelerated methotrexate, vinblastine, doxorubicin, and cisplatin (AMVAC) as neoadjuvant chemotherapy for patients with muscle-invasive transitional cell carcinoma of the bladder. Cancer. 2012; 118: 3920-7.

\title{
Sequenztherapie beim Urothelkarzinom
}

Die neoadjuvante Chemotherapie (CT) ist zwar Standard bei operativ zugänglichen invasiven Urothelkarzinomen, doch limitieren die Nebenwirkungen von Cisplatin den Einsatz. Eine sequenzielle CT könnte eine Alternative sein.

E inbezogen in die Phase-II-Studie waE ren 65 Patienten mit invasivem Urothelkarzinom und hohem Risiko für eine nicht kurative Resektion. Als Risikofaktoren galten z.B. eine Tumorinvasion in umliegende Organe wie Prostata oder Uterus, eine lymphovaskuläre Invasion oder eine Hydronephrose (T3b und T4a). Die Patienten erhielten neoadjuvant drei Zyklen einer Kombination aus Ifosfamid, Doxorubicin und Gemcitabin (IAG) gefolgt von vier Zyklen einer Kombination von Cisplatin, Gemcitabin und Ifosfamid (CGI).

Das mediane Follow-up lag bei 85,3 Monaten. Die Rate für das 5-JahresGesamtüberleben (OS) betrug 63\% (95\%-Konfidenzintervall [95\%-KI] 52$0,76 \%)$ und die Rate für das krankheits- spezifische Überleben (DSS) $68 \%$ (95\%KI 58-0,81\%). Ein Downstaging auf pT1N0 und niedriger wurde bei $50 \%$ der Patienten nach Zystektomie und bei $60 \%$ der Patienten nach Nephro-Ureterektomie festgestellt. Das Downstaging korrelierte mit dem 5-Jahres-OS (bei pT1N0 oder niedriger $87 \%$, bei pT2pT3aN0 $67 \%$ und bei pT3b oder höher oder Lymphknoten-negativer Erkrankung $27 \%$; $\mathrm{p} \leq 0$,001 für $\mathrm{pT} 1$ oder niedriger vs. pT2 oder höher). Histologische Varianten waren assoziiert mit einer schlechteren 5-Jahres-DSS-Rate (50 vs. $83 \%$ bei reinem Übergangszellkarzinom, $\mathrm{p}=0,02)$. Häufigste Grad-3-Toxizitäten waren Infektionen (38\%), febrile Neutropenien $(22 \%)$ und Mukositiden (18\%). Drei Patienten entwickelten eine Grad-
4-Toxizität (Myokardinfarkt, Thrombozytopenie, Erbrechen). Ein neutropenischer Patient mit Pneumonie verweigerte Antibiotika und verstarb (1 Grad-5Toxizität).

Fazit: Bei fortgeschrittenen HochrisikoUrothelkarzinomen zeigt eine neoadjuvante sequenzielle IAG/CGI-Therapie im historischen Vergleich ein ähnliches Ansprechen wie ein Cisplatin-basiertes Schema. Das Downstaging auf pT1N0 und darunter erhöht die Aussichten auf Heilung deutlich. Periphere Neuropathien oder Hörverluste können mit der Sequenztherapie vermieden werden, der hochdosierte Einsatz von Ifosfamid sollte allerdings spezialisierten Zentren vorbehalten bleiben.

Barbara Kreutzkamp

Siefker-Radtke AO et al. A phase 2 clinical trial of sequential neoadjuvant chemotherapy with ifosfamide, doxorubicin, and gemcitabine followed by cisplatin, gemcitabine, and ifosfamide in locally advanced urothelial cancer. Cancer. 2012; 22. August. [Epub ahead of print] 